\title{
Sliced-Time Computations with Re-scaling for Blowing-Up Solutions to Initial Value Differential Equations
}

\author{
Nabil R. Nassif ${ }^{1}$, Dolly Fayyad ${ }^{2}$, and Maria Cortas $^{3}$ \\ 1 American University of Beirut, Beirut Lebanon \\ 2 Lebanese University, Faculty of Science II, Fanar Lebanon \\ 3 Lebanese University, Computer Center, Museum Square, Beirut Lebanon
}

\begin{abstract}
In this paper, we present a new approach to simulate timedependent initial value differential equations which solutions have a common property of blowing-up in a finite time. For that purpose, we introduce the concept of "sliced-time computations", whereby, a sequence of time intervals (slices) $\left\{\left[T_{n-1}, T_{n}\right] \mid n \geq 1\right\}$ is defined on the basis of a change of variables (re-scaling), allowing the generation of computational models that share symbolically or numerically "similarity" criteria. One of these properties is to impose that the re-scaled solution computed on each slice do not exceed a well-defined cut-off value (or threshold) $S$. In this work we provide fundamental elements of the method, illustrated on a scalar ordinary differential equation $y^{\prime}=f(y)$ where $f(y)$ verifies $\int_{0}^{\infty} f(y) d y<\infty$. Numerical results on various ordinary and partial differential equations are available in [7, some of which will be presented in this paper.
\end{abstract}

\section{$1 \quad$ Introduction}

Let $\Omega$ be an open domain of $\mathbb{R}^{n}$ with boundary $\Gamma$, sufficiently regular. We consider in this work some numerical aspects related to the blow-up in finite time, of the solution of the semi-linear parabolic equation consisting in finding $\left\{T_{b}, u\right\}$ where $0<T_{b}<\infty, u: \bar{\Omega} \times\left[0, T_{b}\right)$ such that:

$$
\begin{array}{r}
u_{t}-\Delta u=f(u), x \in \Omega, 0 \leq t<T_{b}, u(x, 0)=u_{0}(x) \geq 0, \forall x \in \Omega, \\
u(x, t)=0, \forall x \in \Gamma, 0<t<T_{b} .
\end{array}
$$

The function $f$ is such that $f(y)=O\left(y^{p}\right)$ or $f(y)=O\left(e^{k y}\right)$ as $y \rightarrow \infty$ and verifies:

$$
f: \mathbb{R} \rightarrow \mathbb{R}^{+} \text {, with } f \in C^{1}, f \text { and } f^{\prime} \text { strictly monotone increasing on } \mathbb{R}^{+} .
$$

Under (2), there exists a unique solution to (11) such that, if $u_{0} \geq 0$, then $u(., t) \geq 0$, for all $t$ in the time domain of existence for $u$. Furthermore, it is easy to verify that under (2 3 ), then $\int_{a}^{\infty} \frac{1}{f(x)} d x<\infty$ for some $a \geq 0$. In fact, it is 
shown in (4 pp. 55-56), that this condition is necessary for finite blow-up time to problem (11). Thus, the solution $\left\{T_{b}, u\right\}$ is assumed to satisfy a blowing-up property, specifically the existence of a time $T_{b}$, such that $\lim _{t \rightarrow T_{b}^{-}}\|u(., t)\|_{\infty}=\infty$. where the $L^{\infty}$-norm is defined by $\|g\|_{\infty}=\max _{x \in \Omega}|g(x)|$, for all functions $g: \mathbb{R}$ $\rightarrow \mathbb{R}$. Under (2), the blow-up time $T_{b}$, verifies (see [4]), $\int_{0}^{\infty}[f(z)]^{-1} d z<$ $T_{b}<\int_{0}^{\infty}\left[f(z)-\lambda_{1} z\right]^{-1} d z$, with $\delta^{*}=\lambda_{1} \sup \left\{\frac{u}{f(u)}, u \geq 0\right\}<1, u_{0}=, \lambda_{1}$ being the first eigenvalue of the problem $-\Delta \Phi=\lambda \Phi$. As to the points of $\Omega$ where the explosion occurs, Friedman et McLeod ([10]) proved that the set $\mathrm{E}$ of blow up points of (1), form a compact subset $E$ in $\Omega$. In case the domain $\Omega$ satisfies a radial symmetry property around the origin o, and $u_{0}$ is a radial function, then $E$ reduces to a single point which is the origin itself. This property is confirmed by [4] who gives also the asymptotic behavior of the solution when $t \rightarrow T_{b}$. Space discretization of (1D) leads naturally to very stiff solutions that demonstrate rapid growth in a short range of time, particularly when numerical time integration gets close to the blow-up time. This rapid growth imposes major restrictions on the time step. It is easily verified in such case, that even adaptive numerical procedures (available for example in the various ode MATLAB solvers) fail to provide an acceptable approximation to either the solution or to the blow-up time. Thus, our objective in this work is to obtain numerical algorithms for finding an approximation to $u$ and as well an estimate of the blow-up time $T_{b}$, using a new rescaling technique. In previous papers (see [8], 9]) we have implemented a numerical method to solve (11) based on an $\epsilon$-perturbation that transforms the system into an equivalent one that possesses a "mass conservation property" (see [13]). The solution of this resulting problem is known to be global, thus subject to be solved using standard numerical methods for stiff systems. Several authors have attempted to solve numerically finite blow-up time problems. To our knowledge, initial work can be found in [11, 12], when $f(u)=\lambda e^{u}$. The authors compute an approximation to the blow-up time, for several values of $\lambda$, through a semi-discretization of the space variables, then solve the resulting ordinary differential system of equations using Runge-Kutta methods. More sophisticated algorithms based on rescaling techniques have been considered by Chorin [6], and also Berger and Kohn [5] when $f(u)=u^{\gamma}$. Such technique can describe accurately the behavior of the solution near the singularity. However, one of its main disadvantages is a varying mesh width and time step at each point in space-time, linked to the rapid growth in the magnitude of the solution. Consequently, this requires simultaneously the adjustment of boundary conditions to avoid the loss of accuracy far from the singularity. In References [1, [2], the authors analyze the blow-up behavior of semidiscretizations of reaction diffusion equations. More recently, Acosta, Duran and Rossi derived in [3] an adaptive time step procedure for a parabolic problem with a non-linear boundary condition that causes blow-up behavior of the solution. They used also an explicit Runge Kutta scheme on the resulting system of ordinary differential equations, obtained from the semi-discretization of the space variable using a standard piecewise linear finite element method followed by lumping 
the mass matrix. A time rescaling procedure is also introduced with necessary and sufficient conditions for the existence of blow-up for the numerical solution.

Our approach in this paper differs from those just cited in the sense that we attempt to rescale simultaneously the time and space variables, aiming at the generation of a sequence of "slices" of time intervals $\left\{\left[T_{n-1}, T_{n}\right]: n=1,2, \ldots\right\}$, such that on each of these subintervals, the computation of the solution can be easily controlled by a preset threshold (or cut-off) value $S$, avoiding thus overflow in the global computations. We illustrate the method on an elementary differential equation $y^{\prime}=f(y)$ having blow-up under (2). In fact, the technique we are presenting was motivated by an invariance property in the cases when $f(y)=e^{y}$ or $f(y)=y^{p}$. The scalar problem consists in finding $\left\{T_{b}, y\right\}$, where $0<T_{b}<\infty, y:\left[0, T_{b}\right) \rightarrow R$ such that:

$$
\frac{d y}{d t}=f(y), 0<t<T_{b}, y(0)=y_{0}, \lim _{t \rightarrow T_{b}} y(t)=\infty .
$$

In order to determine the first time subinterval $\left[0, T_{1}\right]$, we start by normalizing (3), by introducing the normalizing parameters $\alpha^{(1)}$ and $\beta^{(1)}$ through the change of variables $y(t)=y_{0}+\alpha^{(1)} z(s), t=\beta^{(1)} s$. Thus, the base Model corresponding to (3), is given by:

$$
\frac{d z}{d s}=g_{1}(s)=\frac{\beta^{(1)}}{\alpha^{(1)}} f\left(y_{0}+\alpha^{(1)} z\right), 0<s \leq s_{1}, z(0)=0, z\left(s_{1}\right)=S .
$$

where $s_{1}=\int_{0}^{S} \frac{d z}{g_{1}(z)}$. Stepping up the solution forward, we define the $n^{\text {th }}$ slice by rescaling both variables $y$ and $t$ in the initial value problem

$$
\frac{d y}{d t}=f(y), T_{n-1}<t, y\left(T_{n-1}\right)=y_{n-1},
$$

using the change of variables:

$$
t=T_{n-1}+\beta^{(n)} s, y(t)=y_{n-1}+\alpha^{(n)} z(s)
$$

Thus, the subinterval $\left[T_{n-1}, T_{n}\right]$ is determined recurrently for $n \geq 2$ using the rescaled model:

$$
\frac{d z}{d s}=g_{n}(z), 0<s \leq s_{n}, z(0)=0, z\left(s_{n}\right)=S .
$$

The function $g_{n}($.$) is given by g_{n}(z)=\left[f\left(y_{n-1}\right)+\alpha^{(n)} z\right] \frac{\beta^{(n)}}{\alpha^{(n)}}$, with $s_{n}=\int_{0}^{S} g_{n}(z) d z$. Note that, the base and rescaled models are respectively defined by the parameters: $\left\{g_{1}, s_{1}, g_{1}(0), g_{1}(S)\right\}$ and $\left\{g_{n}, s_{n}, g_{n}(0), g_{n}(S)\right\}$. When $g_{n}=g_{1}$, both the rescaled and base models are identical. Such is the case when $f(y)=e^{y}$ or $f(y)=y^{p}$, with respectively $\alpha_{n}=1 \beta_{n}=e^{-y_{n-1}}$ and $\alpha_{n}=y_{n-1} \beta_{n}=\left(y_{n-1}\right)^{1-p}$. In general, the sequences $\left\{T_{n}\right\}$ and $\left\{y_{n}=y\left(T_{n}\right)\right\}$ verify:

$$
T_{n}=T_{n-1}+\beta^{(n)} s_{n}, y_{n}=y_{n-1}+\alpha^{(n)} S
$$


Determining the sequences $\left\{\alpha^{(n)}\right\}$ and $\left\{\beta^{(n)}\right\}$ appears to be crucial in the implementation of our rescaling method. This will be done in sections 2 and 3 respectively. More precisely, the sequence $\left\{\alpha^{(n)}\right\}$ is determined consistently with the explosive behavior of the solution of the initial value problem (3). In section 3 , we analyze the sequence $\left\{\beta^{(n)}\right\}$ which allows the computation of the sequence $\left\{T_{n}\right\}$, using (5,2), $T_{n}=T_{n-1}+\beta^{(n)} s_{n}$. In this view, we start by defining a "similarity" criterion between the base and rescaled models identified by the parameters:

Definition 1. The rescaled model (6) is said to be similar to the base model (4), if there exists two positive constants $c$ and $C$ independant of $n$, such that:

$$
c g_{1}(0) \leq g_{n}(0) \leq g_{n}(z) \leq g_{n}(S) \leq C g_{1}(S)
$$

The sequences $\left\{\alpha^{(n)}\right\}$ and $\left\{\beta^{(n)}\right.$ are determined as follows.

1. The main criteria on which we rely to determine the adequate values of the parameters $\left\{\alpha^{(n)}\right\}$ is the explosive behavior of the initial problem (3). That is, the sequences $\left\{T_{n}\right\}$ and $\left\{y_{n}\right\}$ generated by (7) should satisfy the blow-up behavior of the solution, $\lim _{n \rightarrow \infty} y_{n}=\infty, y_{n}=y\left(T_{n}\right), \lim _{n \rightarrow \infty} T_{n}=T_{b}$. On that basis and given the estimate $\frac{\alpha^{(n)} S}{f\left(y_{n}\right)} \leq T_{n}-T_{n-1} \leq \frac{\alpha^{(n)} S}{f\left(y_{n-1}\right)}$, the sequence $\left\{\alpha^{n}\right\}$ must verify the necessary conditions: (i) the infinite series $\sum_{n=1}^{\infty} \alpha^{(n)}$ is divergent and (ii) $\lim _{n \rightarrow \infty}\left(\frac{\alpha^{(n)}}{f\left(y_{n}\right)}\right)=0$.

Note that the 2 cases, $\alpha^{(n)}=1$ and $\alpha^{(n)}=y_{n-1}$ (corresponding to $g_{n}=g_{1}$, when respectively, $f(y)=e^{y}$ and $f(y)=y^{p}$ ) verify such necessary conditions. Moreover, the resulting sequences $\left\{y_{n}\right\}$ reveal to be respectively geometric or arithmetic, which significantly simplifies the implementation of the rescaling method. Although conditions (i) and (ii) provide a wide range of choices for the sequence $\left\{\alpha^{(n)}\right\}$ we restrict ourselves to the choices $\alpha^{(n)}=y_{n-1}$ in the case $f(y)=O\left(y^{p}\right)$ and $\alpha^{(n)}=1$ if $f(y)=O\left(e^{y}\right)$.

2. For the sequence $\left\{\beta^{(n)}\right\}$, we rely on the estimate $g_{n}(0) \leq g_{n}(z) \leq g_{n}(S)$ which implies $\frac{S}{g_{n}(S)} \leq s_{n} \leq \frac{S}{g_{n}(0)}, \forall n$. Thus, fixing the bounds on $s_{n}$ can be obtained directly by fixing those of $g_{n}$. Since $g_{n}(0)=\frac{\beta^{(n)}}{\alpha^{(n)}} f\left(y_{n-1}\right)$ and $g_{n}(S)=\frac{\beta^{(n)}}{\alpha^{(n)}} f\left(y_{n}\right)$, hence $g_{n}(z)$ depends directly on the ratio $\frac{\beta^{(n)}}{\alpha^{(n)}}$ and the sequence $\left\{y_{n}\right\}$. Since we cannot fix simultaneously $g_{n}(0)$ and $g_{n}(S)$, we let $r_{n}=\frac{f\left(y_{n}\right)}{f\left(y_{n-1}\right)}=\frac{g_{n}(S)}{g_{n}(0)}$ and choose $g_{n}(0)=g_{1}(0)$, implying that $\beta^{(n)}=\alpha^{(n)} \frac{g_{1}(0)}{f\left(y_{n-1}\right)}=\alpha^{(n)} \frac{\beta^{(1)}}{\alpha^{(1)}} \frac{f(0)}{f\left(y_{n-1}\right)}$. Thus $g_{1}(0) \leq g_{n}(z) \leq g_{1}(0) r_{n}$ implying $\frac{S}{r_{n} g_{1}(0)} \leq s_{n} \leq \frac{S}{g_{1}(0)}$. If $f(y)=O\left(y^{p}\right), \alpha^{(n)}=y_{n-1}$ or $f(y)=$ $O\left(e^{y}\right), \alpha^{(n)}=1$, the sequence $\left\{r_{n}\right\}$ is uniformly bounded and one has similarity between the base and rescaled models according to Definition 1. Furthermore, $\frac{T_{n}-T_{n-1}}{T_{1}}=\beta^{(n)} s_{n} \leq \beta^{(n)} \frac{S}{T_{1} g_{1}(0)}$. 
Thus, re-scaling Algorithm Main features are described by the following steps:

1. Choice of the cut-off value $S$, and of the computational tolerance $\varepsilon_{T o l}$

2. Given $y_{\max }^{f}$ the maximum acceptable argument for $f($.$) in the computa-$ tional environment, verify Compatibility Test to insure no overflows would cause process interruption. When $\alpha^{(n)}=1$, Test is given by: $\left\lceil\frac{1}{S} f^{-1}\left(\frac{S}{\varepsilon_{T o l}}\right)\right\rceil+1<\left\lfloor\frac{y_{\max }^{f}}{S}\right\rfloor$.

3. Using RK4 (or other method) solve the base model to determine $\left[0, T_{1}\right]$ and $y(t)$ on $\left[0, T_{1}\right]$ using cut-off value $S$.

(a) Start the recurrence for $n>1$

(b) Re-scaling the variables $\left(t=T_{n-1}+\beta^{(n)} s y(t)=y_{n-1}+\alpha^{(n)} z(s)\right)$

(c) Specify and program the function $g_{n}$.

(d) Solve the re-scaled model using RK4: Determine $s_{n}$ using cut-off value $S$.

(e) Deduce $T_{n}=T_{n-1}+\beta^{(n)} s_{n}$ and $\mathrm{y}(\mathrm{t})$ on $\left[T_{n-1}, T_{n}\right]$.

(f) End recurrence when $\frac{T_{n}-T_{n-1}}{T_{1}} \leq \varepsilon_{T o l} \cdot n_{S T O P}:=n$

4. Set the approximation blow-up as $T_{b}:=T_{n_{S T O P}}$

5. Compile the global solution $y(t)$ on $\left[0, T_{b}\right]$.

6. Visualize the numerical solutions.

\section{Application to a Blowing-Up Semi-linear Parabolic System}

Applying the method of sliced-time computations on the problem (1) is done through a first step consisting of a semi-discretization in space of this system. This leads to a system of ordinary differential equations. In fact, finding an invariance, or even defining a similarity on (1) seems to be a very complex task. On the other hand, dealing with a a first order semi-discretized system resulting from (11), allows an eventual track to applying the method of slicedtime computations. We introduce some notations.

Definition 2. If $\boldsymbol{V}=\left(V_{1}, V_{2}, \ldots, V_{k}\right)^{T} \in \mathbb{R}^{k}$, then $\boldsymbol{F}(\boldsymbol{V})=\left(f\left(V_{1}\right), f\left(V_{2}\right), \ldots\right.$, $f\left(V_{k}\right)^{T} \in \mathbb{R}^{k}$. On the other hand, $D_{\boldsymbol{V}}$ is the diagonal matrix whose diagonal elements are the components of the vector $V$, i.e. $D_{\boldsymbol{V}}=\operatorname{Diag}(\boldsymbol{V}) \in \mathbb{R}^{k \times k}$.

We choose the finite differences method for discretization. Let $h=\left(h_{1}, h_{2}, \ldots\right.$, $\left.h_{d}\right)^{T} \in \mathbb{R}^{d},\left(|h|=\max _{i}\left|h_{i}\right|\right)$, the discretization parameter. $k=k(h)$ is the number of nodes in $\Omega$ as well as the dimension of the semi-discretized problem. The solution $u$ of (1) being known on the boundaries $\Gamma$, the approached solution $\boldsymbol{U}(t)$ of (8) is only about the nodes in $\Omega . k(h)=O\left(|h|^{-d}\right)$ and $\lim _{|h| \rightarrow 0} k(h)=$ $\infty$. Under such conditions, the $i^{\text {th }}$ component of $U_{i}(t)$ is an approximation of the solution $u$ on the node $x_{i} \in \Omega$ and on the time $t$. Let $u_{i}(t)=u\left(x_{i}, t\right)$, $\forall i=1, \ldots, k$ and let $\boldsymbol{u}(t)=\left\{u_{i}(t): 1 \leq i \leq k\right\}$ the resulting vector of $\mathbb{R}^{k}$. The semi-discretization leads to the following problem:

$$
\frac{d \boldsymbol{U}}{d t}+\boldsymbol{A} \boldsymbol{U}=\boldsymbol{F}(\boldsymbol{U}), \boldsymbol{U}(0)=U_{0},
$$


having $\boldsymbol{U}(t) \in \mathbb{R}^{k}$ and $F: \mathbb{R}^{k} \rightarrow \mathbb{R}^{k}$, and $\boldsymbol{A} \in \mathbb{R}^{k \times k}, \boldsymbol{A}$ is a square matrix representing the standard finite differences discretization corresponding to $-\Delta$ on $\Omega$. An $n^{\text {th }}$ slice $\left[T_{n-1}, T_{n}\right]$ for (8), starts with the initial value problem $\frac{d \boldsymbol{U}}{d t}=-\boldsymbol{A} \boldsymbol{U}+\boldsymbol{F}(\boldsymbol{U}), T_{n-1} \leq t, \boldsymbol{U}\left(T_{n-1}\right)=\boldsymbol{U}_{n-1}$. If we consider the change of variables:

$$
U_{i}(t)=U_{i, n-1}+\alpha_{i}^{(n)} Z_{i}(s), \alpha_{i}^{(n)} \neq 0, \forall i, \forall n, t=T_{n-1}+\beta^{(n)} s, \beta^{(n)} \neq 0 .
$$

Written in matrix, this is equivalent to:

$$
\boldsymbol{U}(t)=\boldsymbol{U}_{n-1}+\boldsymbol{D}_{\alpha^{(n)}} \boldsymbol{Z}(s) .
$$

which gives the following form for the rescaled model:

$$
\frac{d \boldsymbol{Z}}{d s}=-\boldsymbol{A}_{n}(\boldsymbol{Z})+\boldsymbol{G}_{n}(\boldsymbol{Z}), \boldsymbol{Z}(0)=0,\left\|\boldsymbol{Z}\left(s_{n}\right)\right\|_{\infty}=S .
$$

where

$$
\boldsymbol{G}_{n}(\boldsymbol{Z}):=\beta^{(n)} \boldsymbol{D}_{\alpha^{(n)}}^{-1}\left[\boldsymbol{F}\left(\boldsymbol{U}_{n-1}+\boldsymbol{D}_{\alpha^{(n)}} \boldsymbol{Z}\right)\right],
$$

and $\boldsymbol{A}_{n}(\boldsymbol{Z}):=\beta^{(n)} \boldsymbol{D}_{\alpha^{(n)}}^{-1}\left[\boldsymbol{A}\left(\boldsymbol{U}_{n-1}+\boldsymbol{D}_{\alpha^{(n)}} \boldsymbol{Z}\right)\right]$. Using (9) and (10), we have for $s=s_{n}, \boldsymbol{U}_{\boldsymbol{n}}=\boldsymbol{U}\left(\boldsymbol{T}_{\boldsymbol{n}}\right)=\boldsymbol{U}_{\boldsymbol{n}-\mathbf{1}}+\boldsymbol{D}_{\alpha^{(n)}} \boldsymbol{Z}\left(\boldsymbol{s}_{\boldsymbol{n}}\right), T_{n}=T_{n-1}+\beta^{(n)} s_{n}$. For $n=1$, (11) defines the base model. No invariance can be found between the base model and the rescaled model. We will therefore be looking for similarity criteria, with $f($.$) verifying (2). Since our numerical tests are limited to the case$ of a single blow-up point, we assume the explosion of the vector $\boldsymbol{U}(t)$ occurs for the component $i_{0}$, i.e. we assume the existence of $i_{0}$ independent of $h$ such that the solution $\boldsymbol{U}(\boldsymbol{t})$ of (8) verifies $\|\boldsymbol{U}(\boldsymbol{t})\|_{\infty}=\left|U_{i_{0}}(t)\right|$. As for $\left\{\alpha_{i}^{(n)}, 1 \leq i \leq k\right\}$, we discern two cases: (i) $f\left(\right.$.) is exponential order leading to $\alpha_{i}^{(n)}=1, \forall i$. and (ii) $f($.$) is polynomial order in which case we choose \alpha_{i}^{(n)}=U_{i, n-1}, \forall i,\left(\alpha_{i}^{(1)}=1\right.$, lorsque $\left.U_{0}=0\right)$. In fact, (9) added to the last identity lead respectively to $\left\|\boldsymbol{U}_{\boldsymbol{n}}\right\|_{\infty}=\left\|\boldsymbol{U}_{\mathbf{0}}\right\|_{\infty}+n S$ or $\left\|\boldsymbol{U}_{\boldsymbol{n}}\right\|_{\infty}=(1+S)^{n}\left\|\boldsymbol{U}_{\mathbf{0}}\right\|_{\infty}$. Using (9), we have respectively $\left|U_{i, n}\right| \leq\left|U_{i, 0}\right|+n S$, if $\alpha_{i}^{(n)}=1$ and $\left|U_{i, n}\right| \leq(1+S)^{n}\left|U_{i, 0}\right|$, if $\alpha_{i}^{(n)}=U_{i, n-1}$. Both relations are consistent with $\lim _{n \rightarrow \infty}\left\|\boldsymbol{U}_{\boldsymbol{n}}\right\|_{\infty}=\infty$. We aim now to define the similarity for (11). In order to obtain $\left\{\beta^{(n)}\right\}$, we noted that in (11), $\frac{d \boldsymbol{Z}}{d s}$ is the sum of a diffusion term $-\boldsymbol{A}_{n}(\boldsymbol{Z})$, and a reaction term $\boldsymbol{G}_{n}(\boldsymbol{Z})$. Since the reaction term rules the behavior of the solution, $\beta^{(n)}$ will be obtained through a lower similarity property of the form $\left\|\boldsymbol{G}_{n}(\mathbf{0})\right\|_{\infty}=\left\|\boldsymbol{G}_{1}(\mathbf{0})\right\|_{\infty}$. This leads to: $\beta^{(n)}\left\|\boldsymbol{D}_{\alpha^{(n)}}^{-1} \boldsymbol{F}\left(\boldsymbol{U}_{\boldsymbol{n - 1}}\right)\right\|_{\infty}=\beta^{(1)}\left\|\boldsymbol{D}_{\alpha^{(1)}}^{-1} \boldsymbol{F}\left(\boldsymbol{U}_{\mathbf{0}}\right)\right\|_{\infty}$, which can also be written as:

$$
\beta^{(n)}=\beta^{(1)} \frac{\left\|\boldsymbol{D}_{\alpha^{(1)}}^{-1}\right\|_{\infty}\left\|\boldsymbol{F}\left(\boldsymbol{U}_{\mathbf{0}}\right)\right\|_{\infty}}{\left\|\boldsymbol{D}_{\alpha^{(n)}}^{-1}\right\|_{\infty}\left\|\boldsymbol{F}\left(\boldsymbol{U}_{\boldsymbol{n}-\mathbf{1}}\right)\right\|_{\infty}}=\beta^{(1)} \frac{\left\|\boldsymbol{D}_{\alpha^{(n)}}\right\|_{\infty}\left\|\boldsymbol{F}\left(\boldsymbol{U}_{\mathbf{0}}\right)\right\|_{\infty}}{\left\|\boldsymbol{D}_{\alpha^{(1)}}\right\|_{\infty}\left\|\boldsymbol{F}\left(\boldsymbol{U}_{\boldsymbol{n}-\mathbf{1}}\right)\right\|_{\infty}} .
$$

Thus, $\beta^{(n)}=\beta^{(1)} \frac{f\left(U_{i_{0}, 0}\right)}{f\left(U_{i_{0}, n-1}\right)}$ if $\alpha_{i}^{(n)}=1$ and $\beta^{(n)}=\beta^{(1)} \frac{\left|U_{i_{0}, n-1}\right|}{\left|U_{i_{0}, 0}\right|} \frac{f\left(U_{i_{0}, 0}\right)}{f\left(U_{i_{0}, n-1}\right)}$ if $\alpha_{i}^{(n)}=U_{i, n-1}$. Computing $\boldsymbol{Z}(\boldsymbol{s})$ on the $n^{t h}$ slice $\left[T_{n-1}, T_{n}\right]$ gives $\boldsymbol{S}_{n}=\boldsymbol{Z}\left(\boldsymbol{s}_{\boldsymbol{n}}\right)$. 

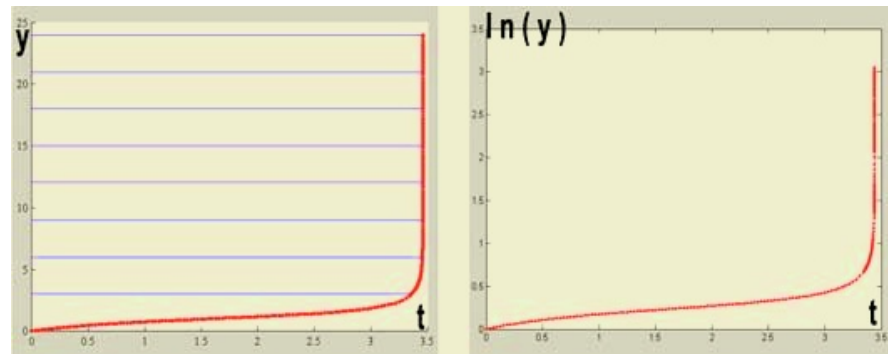

Fig. 1. Variation of the single point of the solution $\boldsymbol{u}$ and of $\ln (\boldsymbol{u})$ against the time t. $\Omega$ is unidimensional, $S=3, h=\frac{1}{4}$ and $\tau=\frac{h^{2}}{2}=\frac{1}{16}$. Note the equal slices due to $\alpha_{i}^{(n)}=1$

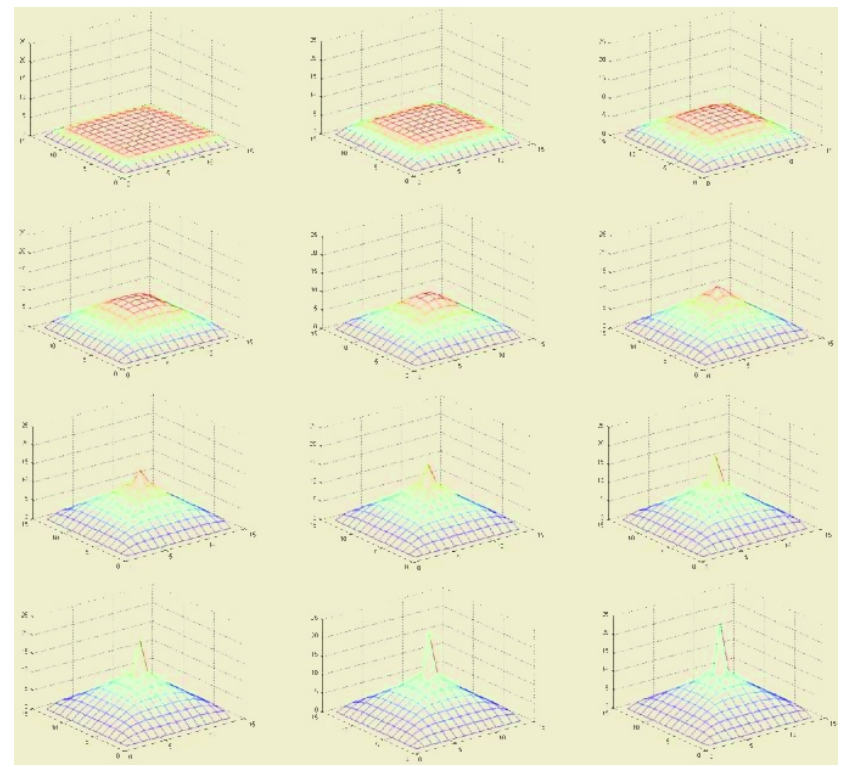

Fig. 2. Variation of the solution $\boldsymbol{u}$ of the problem $\boldsymbol{u}^{\prime}=\Delta \boldsymbol{u}+e^{\boldsymbol{y}}$, over the slices having $\boldsymbol{u}(0)=0, S=2, \Omega$ is bidimensionnel $-1 \leq x \leq 1$ et $-1 \leq y \leq 1$ having $15 \times 15=225$ nodes

Under the validity of $(12)$, we conclude that $\boldsymbol{G}_{n}\left(\boldsymbol{S}_{n}\right)=\beta^{(n)} \boldsymbol{D}_{\alpha^{(n)}}^{-1} \boldsymbol{F}\left(\boldsymbol{U}_{\boldsymbol{n}}\right)$. Consequently, $\quad\left\|\boldsymbol{G}_{n}\left(\boldsymbol{S}_{n}\right)\right\|_{\infty}=\beta^{(n)}\left\|\boldsymbol{D}_{\alpha^{(n)}}^{-1}\right\|_{\infty}\left\|\boldsymbol{F}\left(\boldsymbol{U}_{\boldsymbol{n}-\mathbf{1}}\right)\right\|_{\infty} \frac{\left|f\left(U_{i_{0}, n}\right)\right|}{\left|f\left(U_{i_{0}, n-1}\right)\right|}$ $=\left\|\boldsymbol{G}_{n}(\mathbf{0})\right\|_{\infty} \frac{f\left(U_{i_{0}, n}\right)}{f\left(U_{i_{0}, n-1}\right)}$. Furthermore, since $\boldsymbol{U}_{n}(t)=\boldsymbol{U}_{n-1}+\boldsymbol{D}_{\alpha(n)} \boldsymbol{Z}(s)$, one has: $\left\|\boldsymbol{G}_{n}(\boldsymbol{Z})\right\|_{\infty}=\beta^{(n)}\left\|\boldsymbol{D}_{\alpha^{(n)}}^{-1}\right\|_{\infty}\left\|\boldsymbol{F}\left(\boldsymbol{U}_{\boldsymbol{n}-\mathbf{1}}\right)\right\|_{\infty} \frac{f\left(U_{i_{0}}(t)\right)}{f\left(U_{i_{0}, n-1}\right)}$ and $\left\|\boldsymbol{G}_{n}(\boldsymbol{Z})\right\|_{\infty}=$ $\left\|\boldsymbol{G}_{n}(\mathbf{0})\right\|_{\infty} \frac{f\left(U_{i_{0}}(t)\right)}{f\left(U_{i_{0}, n-1}\right)} \leq\left\|\boldsymbol{G}_{n}(\mathbf{0})\right\|_{\infty} \frac{f\left(U_{i_{0}, n}\right)}{f\left(U_{i_{0}, n-1}\right)}$. Since $f\left(U_{i_{0}}(t)\right) \leq f\left(U_{i_{0}, n}\right)$, one has under the assumptions on $f($.$) added to (13) , \lim _{n \rightarrow \infty} \beta^{(n)}=0$ and $\left\|\boldsymbol{G}_{n}(\boldsymbol{Z})\right\|_{\infty} \leq$ $C\left\|\boldsymbol{G}_{n}(\mathbf{0})\right\|_{\infty}$ if $\frac{f\left(U_{i_{0}, n}\right)}{f\left(U_{i_{0}, n-1}\right)} \leq C, \forall n$. 
A numerical application is conducted on $\frac{\partial u}{\partial t}=\Delta u+e^{u}$. The rescaled models (11) are given by

$$
\begin{aligned}
\frac{d \boldsymbol{Z}}{d s} & =\beta^{(n)} \boldsymbol{D}_{\alpha^{(n)}}^{-1}\left[-\left(\boldsymbol{A} \boldsymbol{U}_{n-1}+\boldsymbol{D}_{\alpha^{(n)}} \boldsymbol{Z}\right)\right] \\
& +\beta^{(n)} \boldsymbol{D}_{\alpha^{(n)}}^{-1}\left[e^{\left(\boldsymbol{U}_{n-1}+\boldsymbol{D}_{\alpha(n)} \boldsymbol{Z}^{\prime}\right)}\right]=-\boldsymbol{A}_{n}(\boldsymbol{Z})+\boldsymbol{G}_{n}(\boldsymbol{Z}), 0<s \leq s_{n},
\end{aligned}
$$

$\boldsymbol{Z}(0)=0,\left\|\boldsymbol{Z}\left(s_{n}\right)\right\|_{\infty}=S$. We pick $\alpha_{i}^{(n)}=1, \forall i, \forall n$, implying $\boldsymbol{D}_{\alpha^{(n)}}=\boldsymbol{I}$, and from (13),$\beta^{(n)}\left\|\boldsymbol{D}_{\alpha^{(n)}}^{-1} e^{\boldsymbol{U}_{\boldsymbol{n}-\mathbf{1}}}\right\|_{\infty}=\beta^{(1)}\left\|\boldsymbol{D}_{\alpha^{(1)}}^{-1} e^{\boldsymbol{U}_{\mathbf{0}}}\right\|_{\infty}$, leading to $\beta^{(n)}=$ $\beta^{(1)} \frac{e^{\left\|U_{0}\right\|_{\infty}}}{e^{\left\|U_{n-1}\right\|_{\infty}}}$. The figures below are few results of numerical experiments conducted on blow-up problems. (See [7]).

\section{References}

1. Abia L.M. , Lopez-Marcos J.C., Martinez J. Blow-up for semidiscretizations of reaction diffusion equations. Appl. Numer. Math. 20(1996)145-156.

2. Abia L.M. , Lopz-Marcos J.C. , Martinez J. On the blow-up time convergence of semidiscretizations of reaction diffusion equations. Appl. Numer. Math. $\underline{26}(1998) 399-414$.

3. Acosta G. , Duran R., Rossi J. An adaptive time step procedure for a parabolic problem with blow-up.

4. Bebernes J., Eberley D. Mathematical Problems from Combustion Theory. Springer-Verlag, (1989).

5. Berger M. , Kohn R. Rescaling algorithm for the numerical calculation of blowingup solutions. Commun. Pure Appl. Math. $\underline{41}(1988) 841-863$.

6. Chorin A. Estimates of intermittency, spectra, and blow-up in developped turbulence. Commun. Pure Appl. Math. 34(1981)853-866.

7. Cortas M. Calcul par tranches pour les équations différentielles à variable temps à caractère explosif. Thesis, Université de Reims, January 2005.

8. Fayyad D. , Nassif N. Approximation of blowing-up solutions to semilinear parabolic equations using "mass-controlled" parabolic systems. Mathematical Modeling and Methods in the Applied Sciences, $\underline{9}$ (1999) pp. 1077-1088.

9. Fayyad D. , Nassif N. On the Numerical Computation of blowing-up solutions for semi-linear parabolic equations. Mathematical Methods in the Applied Sciences,24(2001)pp. 641-657.

10. Friedman A., McLeod B. Blowup of positive solutions of semilinear heat equations. Indiana Univ. Math. J 34 (1985) pp 425-447.

11. Kapila A. K. Reactive-diffusive system with Arrhenius kinetics: Dynamics of ignition. SIAM J. Appl. Math. $\underline{39}$ (1980)21-36.

12. Kassoy D., Poland J. The induction period of a thermal explosion in a gas between infinite parallel plates. Combustion and Flame $\underline{50}$ (1983)259-274.

Crandall M. et al. , Academic Press(1987)159-201.

13. Pierre M., Schmitt D. Blow-up in reactio-diffusion systems with dissipation of mass. Rapport INRIA, $\underline{2652}(1995)$. 